\title{
Periodic Mesoporous Organosilica Functionalized with Sulfonic Acid Groups as Acid Catalyst for Glycerol Acetylation
}

\author{
Els De Canck ${ }^{1}$, Inmaculada Dosuna-Rodríguez ${ }^{2}$, Eric M. Gaigneaux ${ }^{2}$ and Pascal Van Der Voort ${ }^{1, *}$ \\ 1 Center for Ordered Materials, Organometallics \& Catalysis (COMOC), Department of Inorganic \\ and Physical Chemistry, Ghent University, Krijgslaan 281, Building S3, Ghent B-9000, Belgium; \\ E-Mail: els.decanck@ugent.be \\ 2 Institute of Condensed Matter and Nanosciences (IMCN)_Division "MOlecules, Solids and \\ reactiviTy-MOST", Université catholique de Louvain, Croix du Sud 2/L7.05.17, \\ Louvain-la-Neuve B-1348, Belgium; E-Mails: dosuna.inma@gmail.com (I.D.R.); \\ eric.gaigneaux@uclouvain.be (E.M.G.) \\ * Author to whom correspondence should be addressed; E-Mail: pascal.vandervoort@ ugent.be; \\ Tel.: +32-9-264-44-42; Fax: +32-9-264-49-83.
}

Received: 11 July 2013; in revised form: 1 August 2013 / Accepted: 13 August 2013 / Published: 16 August 2013

\begin{abstract}
A Periodic Mesoporous Organosilica (PMO) functionalized with sulfonic acid groups has been successfully synthesized via a sequence of post-synthetic modification steps of a trans-ethenylene bridged PMO material. The double bond is functionalized via a bromination and subsequent substitution obtaining a thiol functionality. This is followed by an oxidation towards a sulfonic acid group. After full characterization, the solid acid catalyst is used in the acetylation of glycerol. The catalytic reactivity and reusability of the sulfonic acid modified PMO material is investigated. The catalyst showed a catalytic activity and kinetics that are comparable with the commercially available resin, Amberlyst-15, and furthermore our catalyst can be recycled for several subsequent catalytic runs and retains its catalytic activity.
\end{abstract}

Keywords: Periodic Mesoporous Organosilica; sulfonic acid groups; catalysis; glycerol acetylation 


\section{Introduction}

The discovery of Periodic Mesoporous Organosilicas (PMOs) [1-3] with organic bridging groups incorporated in their silica framework has been the start of a fascinating research area which provides materials with huge potential [4-6]. Different organic bridges have been employed for very diverse applications, such as heterogeneous catalysts [7,8], bio-sensors [9,10], chromatographic packing materials [11,12], low-k materials [13,14], adsorbents of pollutants [15] and controlled drug delivery systems [16-19]. PMOs are highly porous materials with large specific surface areas, pore volumes and narrow pore size distributions. Furthermore, they exhibit a high thermal and mechanical stability [20-22], especially in comparison with other porous silica materials [23]. This type of material is synthesized with structure directing agents such as the non-ionic triblock copolymer P123. Around this template, a silica source is condensed in basic or acid aqueous environment. Usually, an organo bis-silane $\left(\mathrm{R}^{\prime} \mathrm{O}\right)_{3}-\mathrm{Si}-\mathrm{R}-\mathrm{Si}-\left(\mathrm{OR}^{\prime}\right)_{3}$ is used where $\mathrm{R}$ represents the organic bridging group and $\mathrm{R}^{\prime}$ usually a methyl or ethyl group. Already many reports have appeared on different bridging groups (R) like phenylene, ethylene, ethenylene and ethylbenzene but also more complex and flexible organic functionalities have been described. Furthermore the bridging group can be modified to fine-tune the material for a specific application such as solid acid catalysis [4].

Concerning this topic, some very promising results have already been published regarding the incorporation of an acid functionality such as a sulfonic acid group and its catalytic activity. Several diverse methods have been applied to prepare sulfonic acid containing PMO materials. These strategies include the direct sulfonation of the phenylene bridge [24-26], as first attempted by Inagaki et al. [27], and the cocondensation of an organo bis-silane with (3-mercaptopropyl) trimethoxysilane (MPTMS) [28-31] followed by an oxidation of the thiol functionality. The latter can also be achieved by the in-situ oxidation of the thiol functionality by the addition of $\mathrm{H}_{2} \mathrm{O}_{2}$ during the cocondensation process of tetraethoxyorthosilicate (TEOS) and MPTMS [32,33]. Other silanes have been used in cocondensation processes with an organo bis-silane such as 2-(4-chlorosulfonylphenyl)-trimethoxysilane [34] and perfluorinated alkylsulfonic acid silanes [35-37].

In the specific case of ethenylene bridged PMOs, $-\mathrm{SO}_{3} \mathrm{H}$ moieties can be acquired by the direct sulfonation of the $\mathrm{C}=\mathrm{C}$ bond [38]. However, the sulfonic acid group can detach from the material, depending on the environment used during catalysis. Another explored route is the use of a Diels Alder reaction where the ethene bond acts as dienophile. Kondo et al. $[39,40]$ described the cycloaddition of the ethene bond with benzocyclobutene and subsequently the resulting phenylene moiety is sulfonated to obtain a heterogeneous catalyst. These authors tested this material for several catalytic reactions (esterification of acetic acid with ethanol, the Beckmann and pinacole-pinacolone rearrangement) and the catalyst showed excellent conversion results. This Diels Alder process has been further used to expand the functionalization possibilities [41].

Another example of modifying the surface of an ethenylene bridged PMO material has been reported by the research group of Kaliaguine [42]. First, the surface silanols were end-capped with hexamethyldisilazane after which a Friedel-Crafts alkylation with benzene was made, and subsequently the benzene moiety was sulfonated with concentrated sulfuric acid. The $-\mathrm{SO}_{3} \mathrm{H}$ containing material exhibited a high catalytic activity in the self-condensation of heptanal. 
In this study, the pure trans-ethenylene bridged PMO material [43] is chosen as support material. Thiol functionalities were incorporated according to a procedure previously described by our research group [44] and subsequently oxidized in order to obtain $-\mathrm{SO}_{3} \mathrm{H}$. This material was thoroughly characterized and the solid acid was tested in the acetylation of glycerol. Furthermore, the reusability of this catalyst was investigated.

\section{Results and Discussion}

Starting from the trans-ethenylene bridged PMO (EP), a sulfonic acid modified PMO material is prepared, characterized and its catalytic activity is studied in the acetylation of glycerol. A general overview of the preparation method, including the starting material, is presented in Figure 1. Firstly, an ethenylene bridged Periodic Mesoporous Organosilica is synthesized starting from E-1,2-bis(triethoxysilyl)ethene (Figure 1, pathway A) $[43,45]$.

Figure 1. Summary of the synthetic pathways followed in this study. (A) Preparation of trans-ethenylene bridged Periodic Mesoporous Organosilica [EP]; (B) Bromination of EP [BEP]; (C) Substitution of the bromine with Grignard reagent of 3-chloro-1-propanethiol $\left[\mathrm{EP}-\left(\mathrm{CH}_{2}\right)_{3}-\mathrm{SH}\right]$ and $(\mathbf{D})$ Oxidation with sulfuric acid $\left[\mathrm{EP}-\left(\mathrm{CH}_{2}\right)_{3}-\mathrm{SO}_{3} \mathrm{H}\right]$.

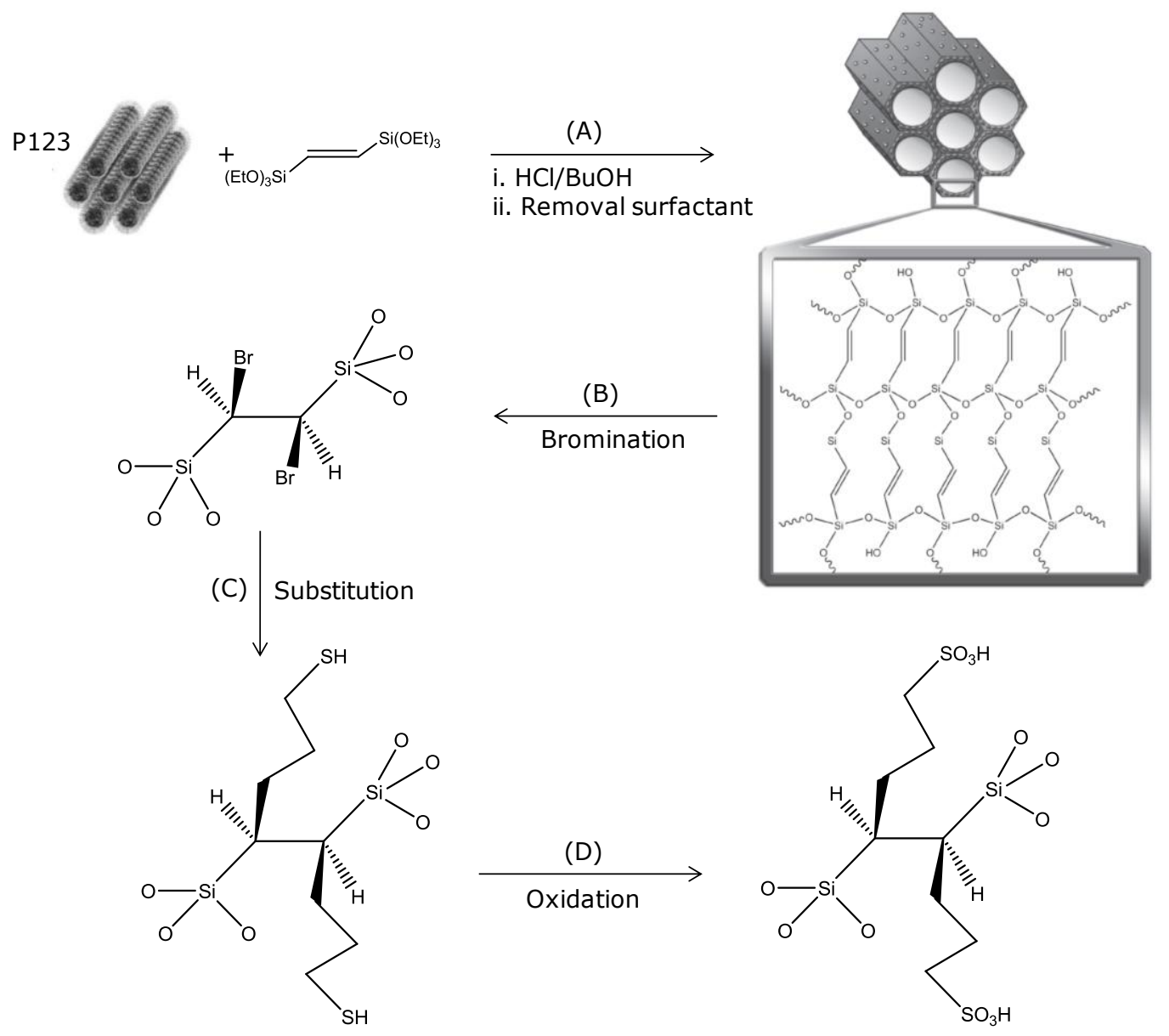

Afterwards, the ethene bridge can be further functionalized to incorporate sulfonic acid groups in the material. When pathway B is followed, the material EP is firstly brominated and subsequently the 
bromine can be substituted via an SN2 reaction by the Grignard reagent of 3-chloro-1-propanethiol (Figure 1, pathway C). A material with a propylthiol group is obtained $\left(\mathrm{EP}-\left(\mathrm{CH}_{2}\right)_{3}-\mathrm{SH}\right)[44,46]$. Oxidizing the thiol moiety is performed with $\mathrm{H}_{2} \mathrm{SO}_{4}$ or other oxidizing agents such as $\mathrm{HNO}_{3}$ [28-31] or $\mathrm{H}_{2} \mathrm{O}_{2}$ [25,47,48]. In this study, a treatment of $\mathrm{EP}-\left(\mathrm{CH}_{2}\right)_{3}-\mathrm{SH}$ with sulfuric acid and a thorough washing step was selected by which it converted the $-\mathrm{SH}$ into a $-\mathrm{SO}_{3} \mathrm{H}$ group (Figure 1, pathway D). This route resulted in the material $\mathrm{EP}-\left(\mathrm{CH}_{2}\right)_{3}-\mathrm{SO}_{3} \mathrm{H}$.

\subsection{Characterization of the Solids}

Nitrogen sorption measurements were performed to examine the porosity of the different materials obtained by the reaction pathway shown in Figure 1 (EP, BEP, EP- $\left(\mathrm{CH}_{2}\right)_{3}-\mathrm{SH}$ and $\left.\mathrm{EP}-\left(\mathrm{CH}_{2}\right)_{3}-\mathrm{SO}_{3} \mathrm{H}\right)$. The nitrogen adsorption and desorption isotherms are shown in Figure 2. The type IV isotherms with the condensation step at relative pressures between 0.55 and 0.75 and the H1 hysteresis of the solids clearly indicate that the materials are mesoporous and possess cylindrical pores with a narrow pore size distribution.

Figure 2. The nitrogen adsorption and desorption isotherms (a) and pore size distributions (b) of (A) EP; (B) BEP; (C) EP- $\left(\mathrm{CH}_{2}\right)_{3}-\mathrm{SH}$ and (D) $\mathrm{EP}-\left(\mathrm{CH}_{2}\right)_{3}-\mathrm{SO}_{3} \mathrm{H}$. The isotherms of $\mathrm{BEP}, \mathrm{EP}-\left(\mathrm{CH}_{2}\right)_{3}-\mathrm{SH}$ and $\mathrm{EP}-\left(\mathrm{CH}_{2}\right)_{3}-\mathrm{SO}_{3} \mathrm{H}$ are vertically offset for clarity by 350,700 and $900 \mathrm{~mL}$ (STP) $\mathrm{g}^{-1}$ (Standard Temperature and Pressure), respectively.

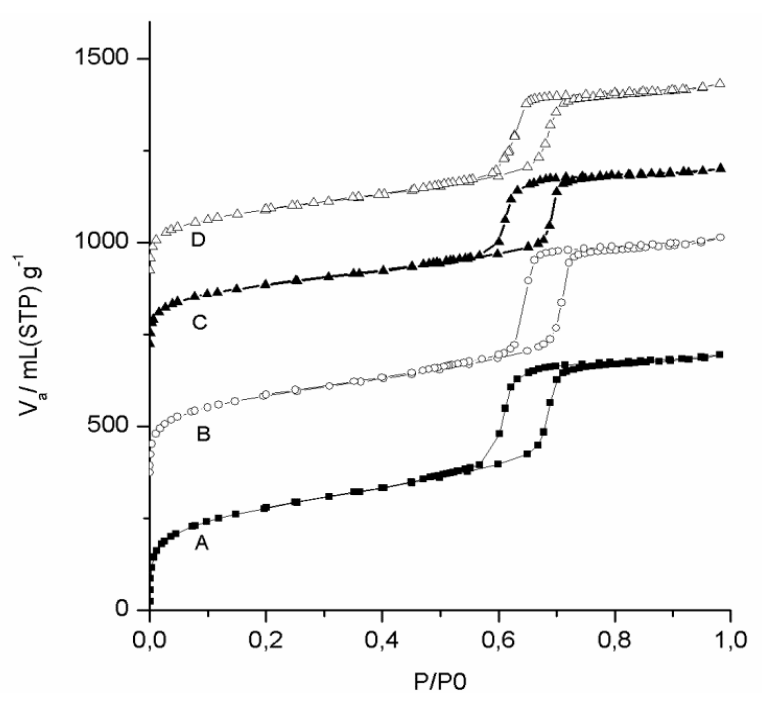

(a)

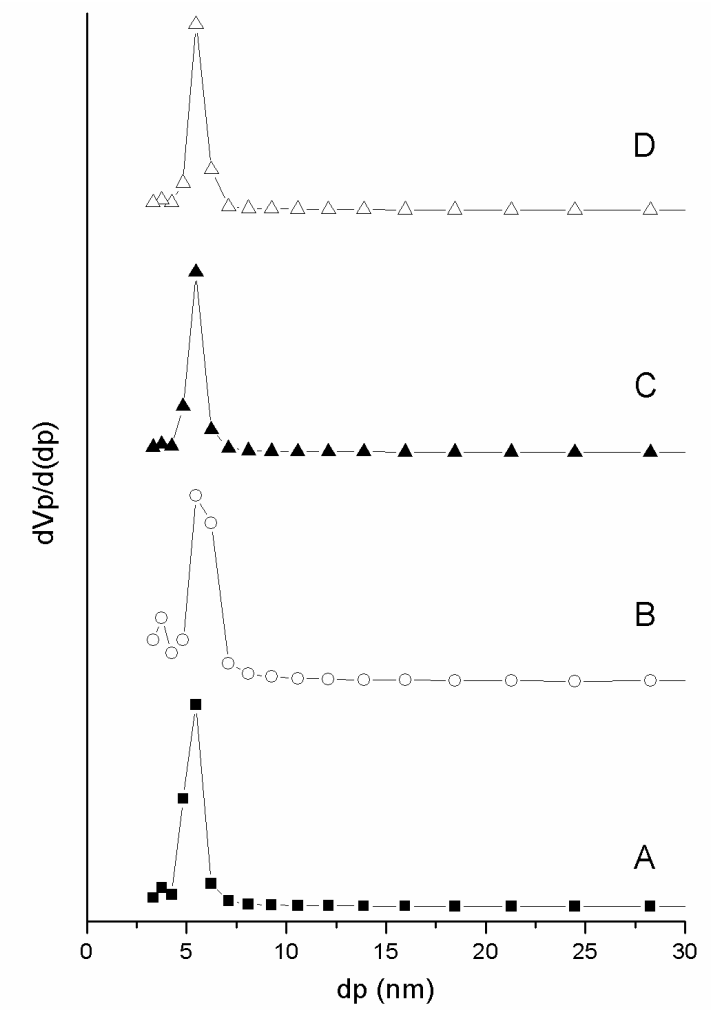

(b)

A summary of the properties of these materials is shown in Table 1. The materials exhibit high specific surface areas $\left(\mathrm{S}_{\mathrm{BET}}\right)$ ranging from 850 to $523 \mathrm{~m}^{2} \mathrm{~g}^{-1}$ and large total pore volumes around $0.84 \mathrm{~mL} \mathrm{~g}^{-1}$. The $\mathrm{S}_{\mathrm{BET}}$ decreases when the material is functionalized due to the decoration of the pore walls with the 
bromine and later on with the propylthiol functionality but also due to the overall weight gain of the functionalized materials. The pore diameter of all the materials lies in the range of 6 to $5 \mathrm{~nm}$. Only a minor shift to smaller pore diameters and a slight broadening of the pore size distribution is observed (Figure 2 and Table 1). The structural characteristics of the commercially available resin Amberlyst-15 are also presented in Table 1 for comparison. This ethenylbenzenesulfonic acid polymer is a strong acid ion exchange resin with unordered macropores. The material is also prone to swelling.

Table 1. Overview of the structural characteristics of the materials compared in this study.

\begin{tabular}{ccccc}
\hline Sample & Path & $\left.\mathbf{S}_{\mathbf{B E T}} \mathbf{a}^{\mathbf{a}} \mathbf{m}^{\mathbf{2}} \mathbf{g}^{\mathbf{- 1}}\right)$ & $\mathbf{V}_{\mathbf{p}}{ }^{\mathbf{b}}\left(\mathbf{m L} \mathbf{g}^{\mathbf{- 1}}\right)$ & $\mathbf{d}_{\mathbf{p}}{ }^{\mathbf{c}}(\mathbf{n m})$ \\
\hline EP & A & 850 & 1.03 & 5.8 \\
BEP & B & 663 & 0.84 & 5.6 \\
$\mathrm{EP}-\left(\mathrm{CH}_{2}\right)_{3}-\mathrm{SH}$ & $\mathrm{C}$ & 523 & 0.59 & 5.3 \\
$\mathrm{EP}-\left(\mathrm{CH}_{2}\right)_{3}-\mathrm{SO}_{3} \mathrm{H}$ & $\mathrm{D}$ & 688 & 0.72 & 5.4 \\
Amberlyst-15 & - & 50 & - & 300 \\
\hline
\end{tabular}

Notes: ${ }^{a}$ Surface area calculated via the Brunauer-Emmett-Teller (BET) model; ${ }^{\mathrm{b}}$ Total pore volume at $\mathrm{P} / \mathrm{P}_{0}=0.98 ;{ }^{\mathrm{c}}$ Pore diameter calculated via the Barrett-Joyner-Halenda (BJH) plot.

The XRD patterns of the materials in Figure 3 reveal three well-resolved signals originating from the low angle (100) and second-order (110) and (200) reflections. This evidently indicates that the materials possess a 2D-hexagonal ordered structure and thus retain their $P 6 \mathrm{~mm}$ space group ordering throughout the syntheses. Only a slight broadening can be observed at the patterns of sample BEP, $\mathrm{EP}-\left(\mathrm{CH}_{2}\right)_{3}-\mathrm{SH}$ and $\mathrm{EP}-\left(\mathrm{CH}_{2}\right)_{3}-\mathrm{SO}_{3} \mathrm{H}$.

Figure 3. The powder X-ray diffraction patterns of (A) EP; (B) BEP; $(\mathbf{C}) \mathrm{EP}-\left(\mathrm{CH}_{2}\right)_{3}-\mathrm{SH}$; and (D) $\mathrm{EP}-\left(\mathrm{CH}_{2}\right)_{3}-\mathrm{SO}_{3} \mathrm{H}$.

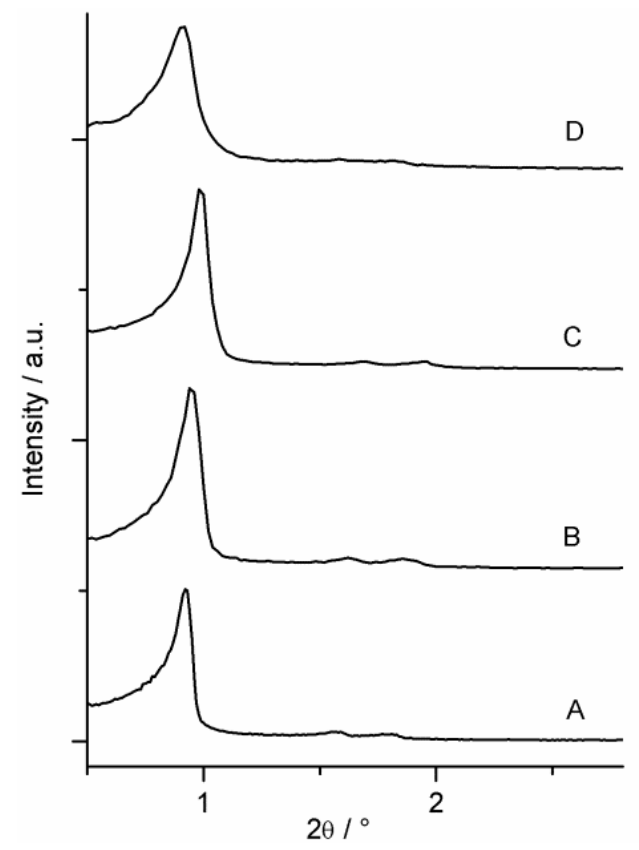

It is quite remarkable that all the materials discussed in this study, show outstanding structural stability. The materials retain porosity and ordering after three consecutive reactions as can be seen 
from the nitrogen sorption and XRD data. These results also confirm the reported stability of Periodic Mesoporous Organosilicas [20,23].

Table 2 presents an overview of the chemical characterization of the solids after the different synthetic procedures. The bromination of the ethene bridge is a very straightforward reaction and approximately $25 \%-30 \%$ of the double bonds are brominated as the remaining fraction of double bonds are buried inside the walls and are unavailable for further reaction [45]. The subsequent substitution with the Grignard reagent results in thiol functionalities. The amount of thiol functionalities is determined using a silver titration [44,46]. After oxidation of the thiol groups using sulfuric acid and a thorough washing step, a total amount of $0.60 \mathrm{mmol} \mathrm{H}^{+}$per gram of material has been observed. This also includes the intrinsic acidity of the PMO material originating from the surface silanols ( $\sim 0.15 \mathrm{mmol} \mathrm{g}^{-1}$ ), as we described earlier [49]. It is clear that the conversion of the thiol containing PMO into the sulfonic acid containing-material has occurred via the oxidation process. This is also confirmed by Raman spectroscopy by the appearance of two signals in the region between 1160 and $1190 \mathrm{~cm}^{-1}$ (See figure S1 in supplementary information). Also, the thiol titration after oxidation showed a zero concentration of remaining thiol groups. Amberlyst-15 exhibits a high acidity of $4.7 \mathrm{mmol} \mathrm{H}^{+} \mathrm{g}^{-1}$.

Table 2. Overview of the chemical characteristics of the materials compared in this study.

\begin{tabular}{ccc}
\hline Sample & Functionality & mmol g $^{-1}$ \\
\hline $\mathrm{BEP}$ & $-\mathrm{Br}^{\mathrm{a}}$ & 2.39 \\
$\mathrm{EP}-\left(\mathrm{CH}_{2}\right)_{3}-\mathrm{SH}$ & $-\mathrm{SH}^{\mathrm{b}}$ & 0.40 \\
$\mathrm{EP}-\left(\mathrm{CH}_{2}\right)_{3}-\mathrm{SO}_{3} \mathrm{H}$ & $-\mathrm{SO}_{3} \mathrm{H}^{\mathrm{c}}$ & $0.60^{\mathrm{d}}$ \\
\hline
\end{tabular}

Notes: ${ }^{\mathrm{a}}$ Determined gravimetrically; ${ }^{\mathrm{b}}$ Determined via silver titration; ${ }^{\mathrm{c}}$ Determined via acid/base titration;

${ }^{\mathrm{d}}$ The deviation between the amount of thiols and total acidity is due to the acidity of the surface silanols.

\subsection{Catalytic Experiments and Recyclability}

The catalytic ability of the sulfonic acid functionalized PMO material has been explored for an esterification reaction, i.e., the glycerol acetylation reaction (Figure 4). The activity of EP- $\left(\mathrm{CH}_{2}\right)_{3}-\mathrm{SO}_{3} \mathrm{H}$ is compared with a commercially available catalyst Amberlyst-15 and moreover the catalysts' reusability is explored.

Figure 4. The esterification reaction: the acetylation of glycerol with the formation of glycerol monoacetate, glycerol diacetate and glycerol triacetate.

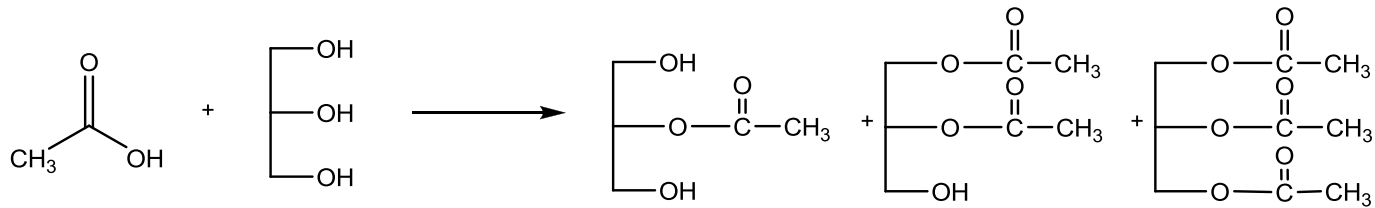

In this study the esterification of glycerol is probed due to its economic importance. Glycerol is an important by-product of first generation biodiesel and is produced in a relative large quantity [50]. This overproduction of glycerol can be used in order to develop second generation biodiesel which uses glycerol as a raw product. As carboxylic acid, acetic acid is probed as shown in the general reaction 
(Figure 4). Three products may in principle be obtained from this reaction: glycerol monoacetate (MAG), glycerol diacetate (DAG) and glycerol triacetate (TAG). However, experimentally, only MAG $(\sim 94 \%)$ and DAG $(\sim 6 \%)$ are formed using the specific catalytic conditions described in the experimental part [51].

The catalytic activity of $\mathrm{EP}-\left(\mathrm{CH}_{2}\right)_{3}-\mathrm{SO}_{3} \mathrm{H}$ for the esterification of acetic acid with glycerol is presented in Figure 5 where the total acetylation yield is shown as a function of time. The total acetylation yield is defined according to the equation below:

$$
\text { Yield }_{\tau}(\%)=\frac{[P]_{t}}{[H A C]_{0}} \frac{v_{H A C}}{v_{p}} \times 100
$$

where $[P]_{\mathrm{t}}$ and $[H A c]_{0}$ represent the product and acetic acid concentration at a certain reaction time and at $t=0$, respectively. Furthermore, $v_{H A c}$ and $v_{P}$ represent the stoichiometric coefficients of the acetic acid and the ester formed, i.e., 1 for mono-substituted, 2 for di-substituted and 3 for fully substituted products, respectively. Also, as acetic acid contains acid protons which can induce a self-catalyzed process, the reaction in absence of any solid catalyst was monitored. Corresponding data are shown in Figure 5. It is clear that the sulfonated PMO possesses a significant catalytic activity with a yield of almost $80 \%$ for this reaction after $\sim 300 \mathrm{~min}$; whereas the blank test (without any solid catalyst involved) yielded only $\sim 50 \%$ of esters after $\sim 300 \mathrm{~min}$. The conversion of Amberlyst-15 is shown in the same figure. Comparing the two materials clearly shows that $\mathrm{EP}-\left(\mathrm{CH}_{2}\right)_{3}-\mathrm{SO}_{3} \mathrm{H}$ exhibits a similar catalytic activity as Amberlyst-15, which is a well-performing catalyst in this type of reaction.

Figure 5. The total acetylation yield for the catalytic reaction with $\mathrm{EP}-\left(\mathrm{CH}_{2}\right)_{3}-\mathrm{SO}_{3} \mathrm{H}$ and Amberlyst-15. Also the blank reaction is represented for clarity. A catalyst loading of $0.25 \mathrm{~g}$ per $40 \mathrm{~mL}$ of glycerol was used. The lines are intended as visual aids only.

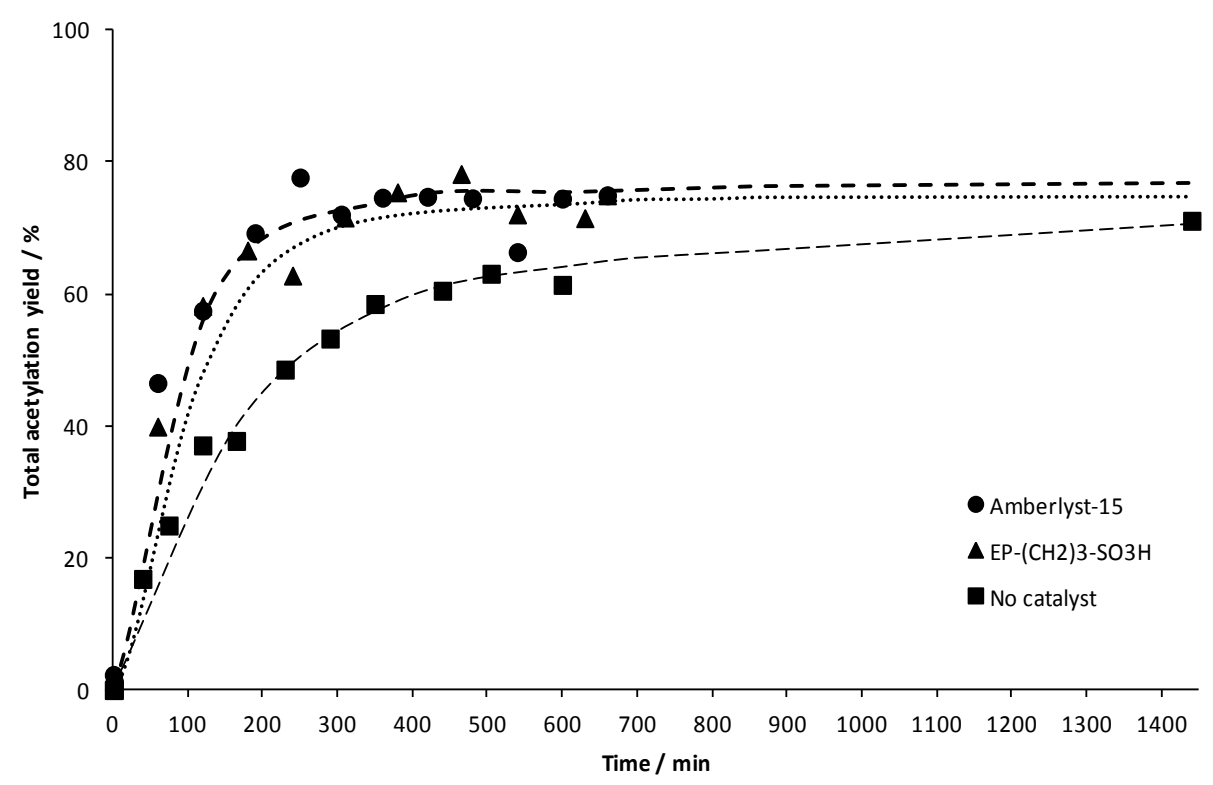

Furthermore, the recyclability of the sulfonic acid containing PMO material is studied for three consecutive runs. First, the initial rate of the catalytic reaction is studied for each run by focusing on the first hour of the acetylation (Figure 6). These experiments are all performed in the same catalytic set-up as the standard catalytic experiment. The solid is filtered after 1 hour and re-used without any 
further treatment in the subsequent run with a fresh reaction medium (run 2); this being performed again for two additional consecutive runs (runs 3 and 4). As one can see from the figure, the material still possesses catalytic activity for the acetylation after four runs. However, recycling of the material in the consecutive runs results in a slight decrease of the initial reaction rate.

Figure 6. Recyclability experiments for $\mathrm{EP}-\left(\mathrm{CH}_{2}\right)_{3}-\mathrm{SO}_{3} \mathrm{H}$ with several runs during the first hour of the reaction. The lines are intended as visual aids only.

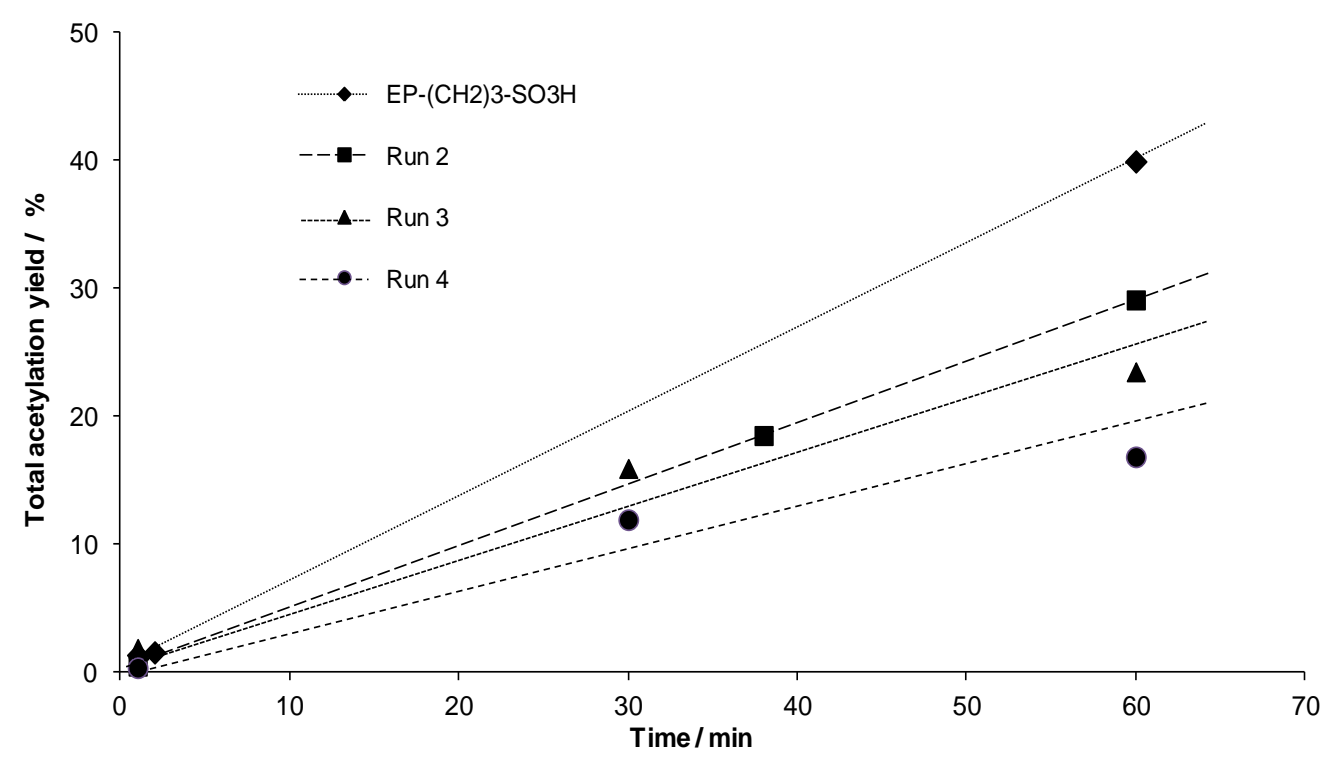

After the last catalytic run, i.e., run 4, the total acetylation yield is monitored for approximately $10 \mathrm{~h} \mathrm{in}$ order to compare it with the acetylation yield of the pristine sulfonated PMO material, $\mathrm{EP}-\left(\mathrm{CH}_{2}\right)_{3}-\mathrm{SO}_{3} \mathrm{H}$ (Figure 7). Although a decrease in the initial reaction rate was observed as already mentioned, at the third consecutive run, the material still reaches the equilibrium after approximately $5 \mathrm{~h}$ and finally results in the same acetylation level as the fresh pristine material.

Figure 7. Recyclability experiment for $\mathrm{EP}-\left(\mathrm{CH}_{2}\right)_{3}-\mathrm{SO}_{3} \mathrm{H}$ : a comparison between the catalytic activity of the pristine material and the third catalytic run. The blank reaction is presented for clarity. The lines are intended as visual aids only.

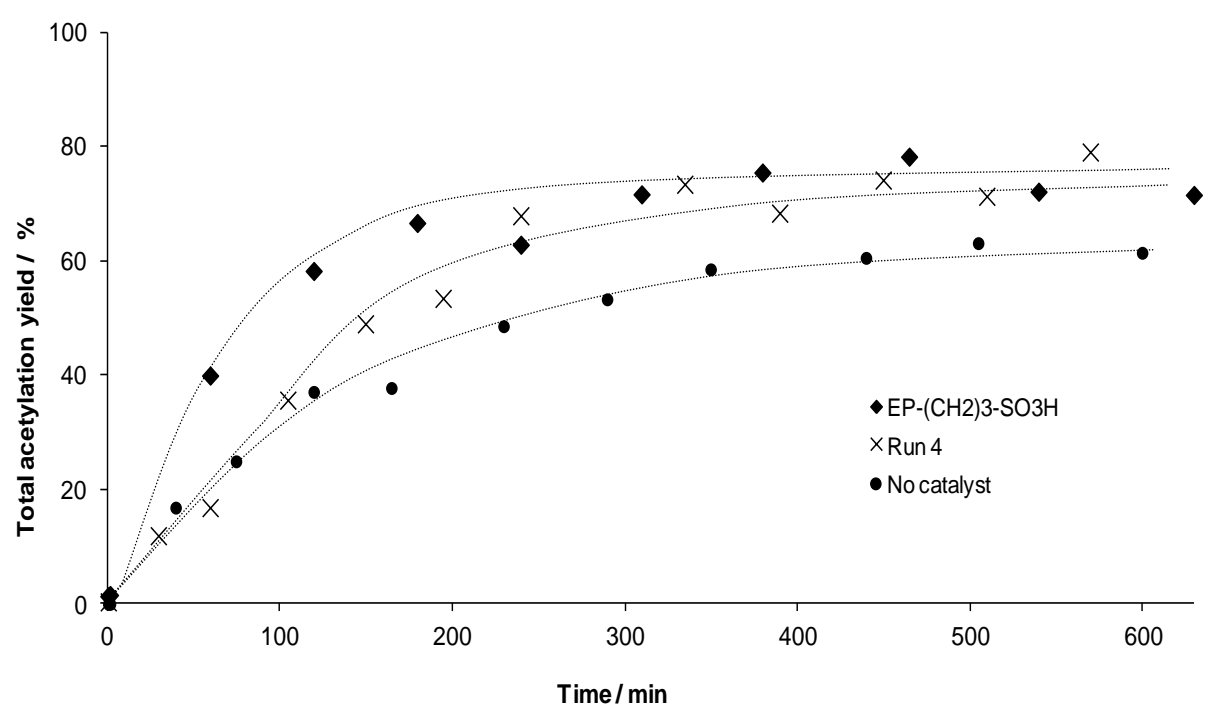


Moreover, additional tests are performed to evaluate the actual heterogeneous character of the observed catalytic activity. Therefore, the solids are removed from the liquid after $1 \mathrm{~h}$ along the first and second runs, and the corresponding recovered solutions are kept under the catalytic conditions to follow the occurrence of a further evolution of the total acetylation yield without solid catalyst in the system anymore. As can be seen from Figure 8, it is clear that the acetylation occurs much slower, i.e., in the range of the blank, when the catalysts are removed of the reaction media, than when the catalyst is maintained in the reactor for the whole test duration.

Figure 8. The total acetylation yield for the $\mathrm{EP}-\left(\mathrm{CH}_{2}\right)_{3}-\mathrm{SO}_{3} \mathrm{H}$, the second and third run. After $60 \mathrm{~min}$ (represented by the vertical line) the liquid is separated from the catalyst and the catalytic activity of the liquid phase of run 2 and 3 is further followed in function of time (open square and triangle). The blank reaction is presented for clarity. The dotted lines are intended as visual aids only.

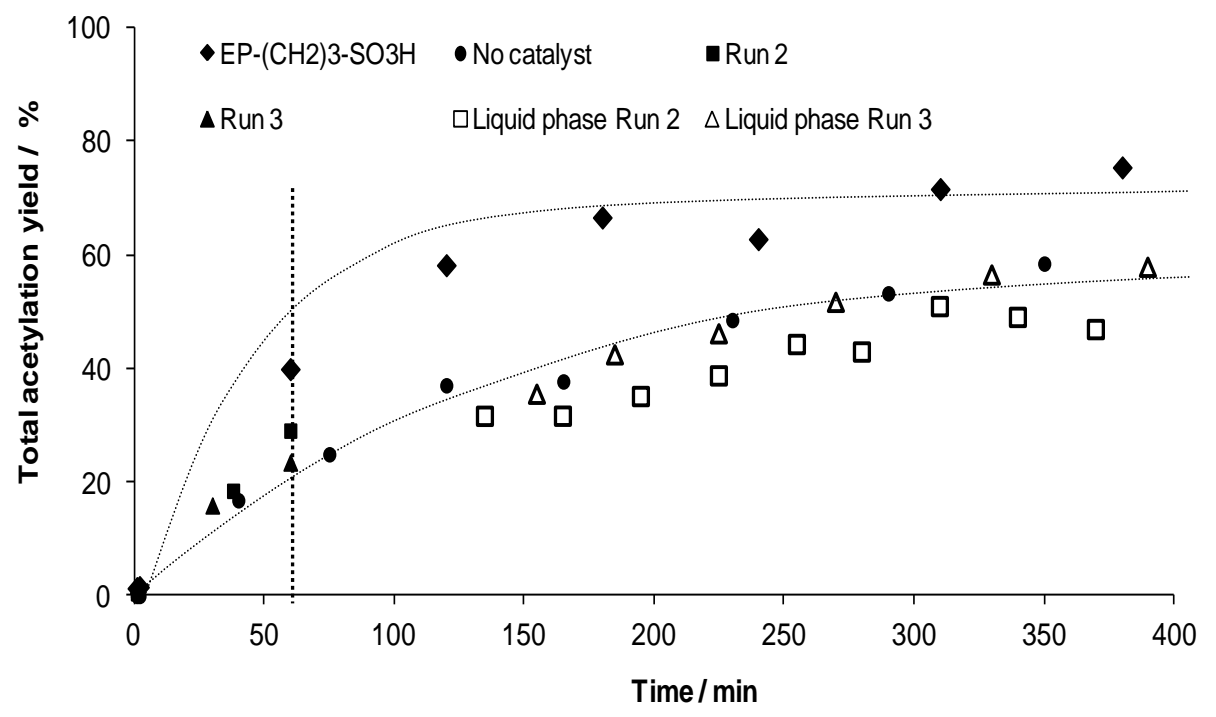

\section{Experimental Section}

\subsection{Materials}

The following reagents were used for the synthesis and characterization: Grubbs' first generation catalyst $\quad\left(\mathrm{PCy}_{3}\right) \mathrm{Cl}_{2} \mathrm{Ru}=\mathrm{CH}-\mathrm{Ph}, \quad$ pluronic $\mathrm{P} 123 \quad\left(\mathrm{PEO}_{20} \mathrm{PPO}_{70} \mathrm{PEO}_{20}\right), \quad$ 1-butanol, bromine, 3-chloro-1-propanethiol (98\%), magnesium turnings (98\%), sodium chloride, potassium thiocyanate, ammonium iron sulfate dodecahydrate, nitric acid (65\%), iron (III) chloride (98\%; anhydrous), toluene (anhydrous), acetonitrile, sulfuric acid $\left(\mathrm{H}_{2} \mathrm{SO}_{4}\right)$, silver nitrate were obtained from Sigma-Aldrich (Bornem, Belgium). Acetone (>99.5\%) was acquired from VWR (Belgium). Tetrahydrofurane (rotidry) and hydrochloric acid (37\%; p.a.) were purchased at Carl Roth (Karlsruhe, Germany). Vinyltriethoxysilane (97\%) was acquired from ABCR (Karlsruhe, Germany). The following reagents were used for the catalytic reaction: acetic acid (puriss. p.a., ACS reagens, $\geq 99.8 \%$, GC/T), glycerol (puriss. p.a., ACS reagens, anhydrous, dist., $\geq 99.5 \%$ ) were purchased from Fluka (Bornem, Belgium). 1-Propanol, 1-heptanol (98\%) and o-xylene (98\%) were obtained from Sigma Aldrich. All chemicals were used as received. 


\subsection{Synthesis of Pure E-1,2-Bis(Triethoxysilyl)Ethene (E-BTSE)}

The diastereoisomerically pure E-isomer of the ethenylene precursor was synthesized according to a procedure described by our research group [43,45]. An amount of $0.0535 \mathrm{~g}$ of the Grubbs' first generation catalyst and $42.95 \mathrm{~mL}$ of vinyltriethoxysilane (VTES) were mixed together under inert atmosphere. The mixture was stirred for $1 \mathrm{~h}$ at room temperature and subsequently refluxed. After $3 \mathrm{~h}$, a distillation was performed to remove remaining VTES. Afterwards, the colorless E-1,2-bis(triethoxysilyl)ethene (E-BTSE) was distilled off under vacuum $(\sim 0.1 \mathrm{~Pa})$.

\subsection{Synthesis of Ethenylene Bridged Periodic Mesoporous Organosilica (Figure 1; Pathway A)}

In a typical synthesis, reported by our group [45], a total amount of $1.00 \mathrm{~g}$ of P123 was placed in a flask and $47.80 \mathrm{~mL}$ of distilled $\mathrm{H}_{2} \mathrm{O}, 3.42 \mathrm{~mL}$ concentrated $\mathrm{HCl}$ and $2.45 \mathrm{~mL} \mathrm{1-butanol} \mathrm{were} \mathrm{added.}$ The solution was stirred for $1.5 \mathrm{~h}$ at room temperature and subsequently $1.86 \mathrm{~mL}$ of E-BTSE was added to the mixture. Next, the mixture was vigorously stirred at $35{ }^{\circ} \mathrm{C}$. After $4 \mathrm{~h}$, the temperature was raised to $90{ }^{\circ} \mathrm{C}$ and aged for $16 \mathrm{~h}$. Afterwards, the mixture was left to cool down, and the white solid was filtered and washed with acetone and distilled water. Finally, P123 was removed via Soxhlet extraction with acetone for $5 \mathrm{~h}$. The white solid, denoted as EP, was dried at $120^{\circ} \mathrm{C}$ under vacuum $(\sim 0.1 \mathrm{~Pa})$.

\subsection{Synthesis of BEP and EP-Propylthiol (Figure 1; Pathways B and C)}

The synthesis of this material was extensively described by our research group $[38,46]$. A certain amount of EP was first brominated with bromine gas under vacuum for $3 \mathrm{~h}$. The resulting material was then dried at $90{ }^{\circ} \mathrm{C}$ for $16 \mathrm{~h}$. Magnesium $(0.74 \mathrm{~g})$, iron(III) chloride $(0.54 \mathrm{~g})$ and dry tetrahydrofuran (THF) $(30 \mathrm{~mL})$ were mixed under inert atmosphere and stirred for $30 \mathrm{~min}$ at $50{ }^{\circ} \mathrm{C}$. Next, 3-chloro-1-propanethiol $(0.22 \mathrm{~mL})$ was added and left to stir for $4 \mathrm{~h}$ at room temperature. Subsequently, the solution was added to $0.70 \mathrm{~g}$ of dry brominated EP. The resulting mixture was stirred for an additional $24 \mathrm{~h}$ at $40{ }^{\circ} \mathrm{C}$. Then, the solid was filtered and washed several times with THF, $2 \mathrm{~mol} \mathrm{~L}{ }^{-1} \mathrm{HCl}, \mathrm{H}_{2} \mathrm{O}$ and acetone. Finally, the material was dried at $90{ }^{\circ} \mathrm{C}$ for $16 \mathrm{~h}$ under vacuum $(\sim 0.1 \mathrm{~Pa})$ and denoted as $\mathrm{EP}-\left(\mathrm{CH}_{2}\right)_{3}-\mathrm{SH}$.

\subsection{Synthesis of EP-Propylsulfonic Acid (Figure 1; Pathway D)}

A volume of $25 \mathrm{~mL} \mathrm{H}_{2} \mathrm{SO}_{4}\left(2.5 \mathrm{~mol} \mathrm{~L}^{-1}\right)$ was added to $0.50 \mathrm{~g}$ of $\mathrm{EP}-\left(\mathrm{CH}_{2}\right)_{3}-\mathrm{SH}$ and stirred at room temperature. After $1 \mathrm{~h}$, the solids were filtered and washed thoroughly with water and acetone. Finally, the material was dried at $90{ }^{\circ} \mathrm{C}$ for $16 \mathrm{~h}$ under vacuum $(\sim 0.1 \mathrm{~Pa})$ and referred to as $\mathrm{EP}-\left(\mathrm{CH}_{2}\right)_{3}-\mathrm{SO}_{3} \mathrm{H}$.

\subsection{Determination of the Amount of Reachable Thiols}

A titration is performed as described in the literature [44]. A solution of silver nitrate with a known concentration is added to $0.050 \mathrm{~g}$ of material and left to stir until the equilibrium is reached. The excess of silver is titrated with potassium thiocyanate and $\mathrm{FeNH}_{4}\left(\mathrm{SO}_{4}\right)_{2} \cdot 12 \mathrm{H}_{2} \mathrm{O}$ in $0.3 \mathrm{~mol} \mathrm{~L}-1 \mathrm{HNO}_{3}$ as indicator. 


\subsection{Determination of the Total Acidity}

The material containing sulfonic acid groups was stirred for $24 \mathrm{~h}$ in $20 \mathrm{~g}$ of $2 \mathrm{~mol} \mathrm{~L}^{-1} \mathrm{NaCl}$. Next, an acid-base titration was achieved with $\mathrm{NaOH}$ and phenolphtaleine as indicator.

\subsection{Esterification of Acetic Acid with Glycerol}

The catalytic reaction was carried out at $105^{\circ} \mathrm{C}$ and at atmospheric pressure in a round bottom flask reactor equipped with a magnetic stirrer. The sulfonic acid containing material was dried prior to use at $105{ }^{\circ} \mathrm{C}$ under vacuum $(\sim 1.33 \mathrm{~Pa})$ for $18 \mathrm{~h}$. The following concentrations of the reagents were used: $100 \mathrm{~g}$ acetic acid/L of glycerol and the catalyst concentration was $6.25 \mathrm{~g} / \mathrm{L}$ of glycerol. First, the catalyst and glycerol are added together to the flask and are brought to reaction temperature. When the temperature is reached, the acetic acid is injected. Before analysis the samples are extracted with 1-heptanol and using $o$-xylene as an internal standard. The total yield of the esters was determined with gas chromatography. After one minute a sample of the reaction mixture is taken and analyzed with gas chromatography. This sample represents the starting point of the catalytic experiment. The recyclability experiments were carried out under identical conditions as the catalytic tests. Therefore, the catalyst was separated from the liquid phase after $1 \mathrm{~h}$ and reused in a consecutive run without further treatment. The liquid phase was kept under reaction conditions and further followed as a function of time. Gas chromatography was performed with a Varian CP-3800 using a Cp-Sil 8CD column (Varian, Palo Alto, CA, USA) with a FID detector.

\subsection{Characterization}

Nitrogen sorption measurements were carried out by a Belsorp-mini II gas analyzer at $77 \mathrm{~K}$. The specific surface area $\left(\mathrm{S}_{\mathrm{BET}}\right)$ was determined by the BET equation $\left(\mathrm{p} / \mathrm{p}_{0}=0.05-0.15\right)$. The pore size distribution was determined from the desorption branch of the isotherm using the Barrett-Joyner-Halenda (BJH) theory. The samples were pretreated at $90{ }^{\circ} \mathrm{C}$ while degassing ( 0.1 Pa). X-ray diffraction (XRD) measurements were conducted with an ARL X'tra X-ray diffractometer of Thermo Scientific (Waltham, MA, USA) equipped with a $\mathrm{Cu} \mathrm{K}_{\alpha 1}$ tube and a Peltier cooled lithium drifted silicon solid stage detector. Raman spectra are recorded using a Raman type FRA106/S spectrometer of Bruker (Karlsruhe, Germany), equipped with a Nd-YAG laser $(\lambda=1064 \mathrm{~nm})$.

\section{Conclusions}

Periodic Mesoporous Organosilica functionalized with sulfonic acid groups has been successfully synthesized and characterized. An ethenylene bridged PMO material was chosen as starting material and this was further post-modified in several steps. A bromination and subsequent substitution reaction was used followed by an oxidation turning the material into a solid acid catalyst. This material has been investigated for the acetylation of glycerol. The material showed an equal activity as Amberlyst-15; the latter being usually considered as the reference most-efficient catalyst material for such kind of reactions. Recyclability experiments showed that the sulfonated Periodic Mesoporous Organosilica is reusable and showed the same total acetylation yield after three runs. Homogeneous tests showed that 
the acetylation of glycerol indeed occurs heterogeneously, suggesting the absence of leaching, e.g., of sulfonate species, in the medium, and thus the high stability of our material under working conditions.

\section{Acknowledgments}

EDC and PVDV thank Ghent University for financial support and Tom Planckaert from the department of Inorganic and Physical Chemistry (Ghent University) for nitrogen sorption and X-ray diffraction measurements. IDR and EMG acknowledge the support of the Région Wallonne (DG06, convention LIGNOFUEL No. 716721).

\section{Conflicts of Interest}

The authors declare no conflict of interest.

\section{References}

1. Inagaki, S.; Guan, S.; Fukushima, Y.; Ohsuna, T.; Terasaki, O. Novel mesoporous materials with a uniform distribution of organic groups and inorganic oxide in their frameworks. J. Am. Chem. Soc. 1999, 121, 9611-9614.

2. Asefa, T.; MacLachan, M.J.; Coombs, N.; Ozin, G.A. Periodic mesoporous organosilicas with organic groups inside the channel walls. Nature 1999, 402, 867-871.

3. Melde, B.J.; Holland, B.T.; Blanford, C.F.; Stein, A. Mesoporous sieves with unified hybrid inorganic/organic frameworks. Chem. Mater. 1999, 11, 3302-3308.

4. Van der Voort, P.; Esquivel, D.; de Canck, E.; Goethals, F.; van Driessche, I.; Romero-Salguero, F.J. Periodic Mesoporous Organosilicas: from simple to complex bridges; A comprehensive overview of functions, morphologies and applications. Chem. Soc. Rev. 2013, 42, 3913-3955.

5. Mizoshita, N.; Tani, T.; Inagaki, S. Syntheses, properties and applications of periodic mesoporous organosilicas prepared from bridged organosilane precursors. Chem. Soc. Rev. 2011, 40, 789-800.

6. Hoffmann, F.; Froba, M. Vitalising porous inorganic silica networks with organic functions-PMOs and related hybrid materials. Chem. Soc. Rev. 2011, 40, 608-620.

7. Sasidharan, M.; Fujita, S.; Ohashi, M.; Goto, Y.; Nakashima, K.; Inagaki, S. Novel synthesis of bifunctional catalysts with different microenvironments. Chem. Commun. 2011, 47, 10422-10424.

8. Ohashi, M.; Kapoor, M.P.; Inagaki, S. Chemical modification of crystal-like mesoporous phenylene-silica with amino group. Chem. Commun. 2008, 7, 841-843.

9. Johnson-White, B.; Zeinali, M.; Shaffer, K.M.; Patterson, C.H.; Charles, P.T.; Markowitz, M.A. Detection of organics using porphyrin embedded nanoporous organosilicas. Biosens. Bioelectron. 2007, 22, 1154-1162.

10. Johnson-White, B.; Zeinali, M.; Malanoski, A.P.; Dinderman, M.A. Sunlight-catalyzed conversion of cyclic organics with novel mesoporous organosilicas. Catal. Commun. 2007, 8, 1052-1056.

11. Huang, L.L.; Lu, J.; Di, B.; Feng, F.; Su, M.X.; Yan, F. Self-assembled highly ordered ethane-bridged periodic mesoporous organosilica and its application in HPLC. J. Sep. Sci. 2011, 34, 2523-2527. 
12. Zhang, Y.P.; Jin, Y.; Yu, H.; Dai, P.C.; Ke, Y.X.; Liang, X.M. Pore expansion of highly monodisperse phenylene-bridged organosilica spheres for chromatographic application. Talanta 2010, 81, 824-830.

13. Goethals, F.; Baklanov, M.R.; Ciofi, I.; Detavernier, C.; van der Voort, P.; van Driessche, I. A new procedure to seal the pores of mesoporous low-k films with precondensed organosilica oligomers. Chem. Commun. 2012, 48, 2797-2799.

14. Goethals, F.; Ciofi, I.; Madia, O.; Vanstreels, K.; Baklanov, M.R.; Detavernier, C.; van der Voort, P.; van Driessche, I. Ultra-low-k cyclic carbon-bridged PMO films with a high chemical resistance. $J$. Mater. Chem. 2012, 22, 8281-8286.

15. Walcarius, A.; Mercier, L. Mesoporous organosilica adsorbents: Nanoengineered materials for removal of organic and inorganic pollutants. J. Mater. Chem. 2010, 20, 4478-4511.

16. Parambadath, S.; Rana, V.K.; Moorthy, S.; Chu, S.W.; Park, S.K.; Lee, D.; Sung, G.; Ha, C.S. Periodic mesoporous organosilicas with co-existence of diurea and sulfanilamide as an effective drug delivery carrier. J. Solid State Chem. 2011, 184, 1208-1215.

17. Parambadath, S.; Rana, V.K.; Zhao, D.Y.; Ha, C.S. $N, N^{\prime}$-diureylenepiperazine-bridged periodic mesoporous organosilica for controlled drug delivery. Microporous Mesoporous Mater. 2011, 141, 94-101.

18. Vathyam, R.; Wondimu, E.; Das, S.; Zhang, C.; Hayes, S.; Tao, Z.M.; Asefa, T. Improving the adsorption and release capacity of organic-functionalized mesoporous materials to drug molecules with temperature and synthetic methods. J. Phys. Chem. C 2011, 115, 13135-13150.

19. Moorthy, M.S.; Park, S.S.; Fuping, D.; Hong, S.H.; Selvaraj, M.; Ha, C.S. Step-up synthesis of amidoxime-functionalised periodic mesoporous organosilicas with an amphoteric ligand in the framework for drug delivery. J. Mater. Chem. 2012, 22, 9100-9108.

20. Goethals, F.; Vercaemst, C.; Cloet, V.; Hoste, S.; van der Voort, P.; van Driessche, I. Comparative study of ethylene- and ethenylene-bridged periodic mesoporous organosilicas. Microporous Mesoporous Mater. 2010, 131, 68-74.

21. Kruk, M.; Jaroniec, M.; Guan, S.Y.; Inagaki, S. Adsorption and thermogravimetric characterization of mesoporous materials with uniform organic-inorganic frameworks. J. Phys. Chem. B 2001, 105, 681-689.

22. Esquivel, D.; Jimenez-Sanchidrian, C.; Romero-Salguero, F.J. Comparison of the thermal and hydrothermal stabilities of ethylene, ethylidene, phenylene and biphenylene bridged periodic mesoporous organosilicas. Mater. Lett. 2011, 65, 1460-1462.

23. Burleigh, M.C.; Markowitz, M.A.; Jayasundera, S.; Spector, M.S.; Thomas, C.W.; Gaber, B.P. Mechanical and hydrothermal stabilities of aged periodic mesoporous organosilicas. J. Phys. Chem. B 2003, 107, 12628-12634.

24. Esquivel, D.; Jimenez-Sanchidrian, C.; Romero-Salguero, F.J. Thermal behaviour, sulfonation and catalytic activity of phenylene-bridged periodic mesoporous organosilicas. J. Mater. Chem. 2011, 21, 724-733.

25. Rac, B.; Hegyes, P.; Forgo, P.; Molnar, A. Sulfonic acid-functionalized phenylene-bridged periodic mesoporous organosilicas as catalyst materials. Appl. Catal. A Gen. 2006, 299, 193-201. 
26. Kapoor, M.P.; Kasama, Y.; Yanagi, M.; Yokoyama, T.; Inagaki, S.; Shimada, T.; Nanbu, H.; Juneja, L.R. Cubic phenylene bridged mesoporous hybrids from allylorganosilane precursors and their applications in Friedel-Crafts acylation reaction. Microporous Mesoporous Mater. 2007, 101, 231-239.

27. Inagaki, S.; Guan, S.; Ohsuna, T.; Terasaki, O. An ordered mesoporous organosilica hybrid material with a crystal-like wall structure. Nature 2002, 416, 304-307.

28. Yang, Q.H.; Kapoor, M.P.; Shirokura, N.; Ohashi, M.; Inagaki, S.; Kondo, J.N.; Domen, K. Ethane-bridged hybrid mesoporous functionalized organosilicas with terminal sulfonic groups and their catalytic applications. J. Mater. Chem. 2005, 15, 666-673.

29. Yang, Q.H.; Kapoor, M.P.; Inagaki, S.; Shirokura, N.; Kondo, J.N.; Domen, K. Catalytic application of sulfonic acid functionalized mesoporous benzene-silica with crystal-like pore wall structure in esterification. J. Mol. Catal. A Chem. 2005, 230, 85-89.

30. Yang, Q.H.; Liu, J.; Yang, J.; Kapoor, M.P.; Inagaki, S.; Li, C. Synthesis, characterization, and catalytic activity of sulfonic acid-functionalized periodic mesoporous organosilicas. J. Catal. 2004, 228, 265-272.

31. Yang, Q.H.; Kapoor, M.P.; Inagaki, S. Sulfuric acid-functionalized mesoporous benzene-silica with a molecular-scale periodicity in the walls. J. Am. Chem. Soc. 2002, 124, 9694-9695.

32. Melero, J.A.; Vicente, G.; Morales, G.; Paniagua, M.; Moreno, J.M.; Roldan, R.; Ezquerro, A.; Perez, C. Acid-catalyzed etherification of bio-glycerol and isobutylene over sulfonic mesostructured silicas. Appl. Catal. A Gen. 2008, 346, 44-51.

33. Melero, J.A.; Bautista, L.F.; Morales, G.; Iglesias, J.; Sanchez-Vazquez, R. Biodiesel production from crude palm oil using sulfonic acid-modified mesostructured catalysts. Chem. Eng. J. 2010, $161,323-331$.

34. Rat, M.; Zahedi-Niaki, M.H.; Kaliaguine, S.; Do, T.O. Sulfonic acid functionalized periodic mesoporous organosilicas as acetalization catalysts. Microporous Mesoporous Mater. 2008, 112, $26-31$.

35. Dube, D.; Rat, M.; Shen, W.; Nohair, B.; Beland, F.; Kaliaguine, S. Perfluorinated alkylsulfonic acid functionalized periodic mesostructured organosilica: A new acidic catalyst. Appl. Catal. A Gen. 2009, 358, 232-239.

36. Dube, D.; Rat, M.; Shen, W.; Beland, F.; Kaliaguine, S. Perfluoroalkylsulfonic acid-functionalized periodic mesostructured organosilica: a strongly acidic heterogeneous catalyst. J. Mater. Sci. 2009, 44, 6683-6692.

37. Shen, W.; Dube, D.; Kaliaguine, S. Alkylation of isobutane/1-butene over periodic mesoporous organosilica functionalized with perfluoroalkylsulfonic acid group. Catal. Commun. 2008, 10, 291-294.

38. De Canck, E.; Vercaemst, C.; Verpoort, F.; van der Voort, P. A New Sulphonic Acid Functionalized Periodic Mesoporous Organosilica as A Suitable Catalyst. In Scientific Bases for the Preparation of Heterogeneous Catalysts: Proceedings of the 10th International Symposium; Gaigneaux, E.M.; Devillers, M.; Hermans, S.; Jacobs, P.A.; Martens, J.A.; Ruiz, P., Eds.; Elsevier Science BV: Amsterdam, The Netherlands, 2010; Volume 175, pp. 365-368.

39. Nakajima, K.; Tomita, I.; Hara, M.; Hayashi, S.; Domen, K.; Kondo, J.N. A stable and highly active hybrid mesoporous solid acid catalyst. Adv. Mater. 2005, 17, 1839-1842. 
40. Nakajima, K.; Tomita, I.; Hara, M.; Hayashi, S.; Domen, K.; Kondo, J.N. Development of highly active $\mathrm{SO}_{3} \mathrm{H}$-modified hybrid mesoporous catalyst. Catal. Today 2006, 116, 151-156.

41. Esquivel, D.; de Canck, E.; Jimenez-Sanchidrian, C.; van der Voort, P.; Romero-Salguero, F.J. Formation and functionalization of surface Diels-Alder adducts on ethenylene-bridged periodic mesoporous organosilica. J. Mater. Chem. 2011, 21, 10990-10998.

42. Dube, D.; Rat, M.; Beland, F.; Kaliaguine, S. Sulfonic acid functionalized periodic mesostructured organosilica as heterogeneous catalyst. Microporous Mesoporous Mater. 2008, $111,596-603$.

43. Vercaemst, C.; Ide, M.; Allaert, B.; Ledoux, N.; Verpoort, F.; van der Voort, P. Ultra-fast hydrothermal synthesis of diastereoselective pure ethenylene-bridged periodic mesoporous organosilicas. Chem. Commun. 2007, 22, 2261-2263.

44. De Canck, E.; Lapeire, L.; de Clercq, J.; Verpoort, F.; van der Voort, P. New ultrastable mesoporous adsorbent for the removal of mercury ions. Langmuir 2010, 26, 10076-10083.

45. Vercaemst, C.; Ide, M.; Wiper, P.V.; Jones, J.T.A.; Khimyak, Y.Z.; Verpoort, F.; van ver Voort, P. Ethenylene-Bridged Periodic Mesoporous Organosilicas: From E to Z. Chem. Mater. 2009, 21, 5792-5800.

46. Gao, Y.; de Canck, E.; Leermakers, M.; Baeyens, W.; van der Voort, P. Synthesized mercaptopropyl nanoporous resins in DGT probes for determining dissolved mercury concentrations. Talanta 2011, 87, 262-267.

47. Hamoudi, S.; Royer, S.; Kaliaguine, S. Propyl- and arene-sulfonic acid functionalized periodic mesoporous organosilicas. Microporous Mesoporous Mater. 2004, 71, 17-25.

48. Hamoudi, S.; Kaliaguine, S. Sulfonic acid-functionalized periodic mesoporous organosilica. Microporous Mesoporous Mater. 2003, 59, 195-204.

49. Ide, M.; El-Roz, M.; de Canck, E.; Vicente, A.; Planckaert, T.; Bogaerts, T.; van Driessche, I.; Lynen, F.; van Speybroeck, V.; Thybault-Starzyk, F.; van der Voort, P. Quantification of silanol sites for the most common mesoporous ordered silicas and organosilicas: total versus accessible silanols. Phys. Chem. Chem. Phys. 2013, 15, 642-650.

50. Nigam, P.S.; Singh, A. Production of liquid biofuels from renewable resources. Prog. Energy Combust. Sci. 2011, 37, 52-68.

51. Dosuna-Rodriguez, I.; Gaigneaux, E.M. Glycerol acetylation catalysed by ion exchange resins. Catal. Today 2012, 195, 14-21.

(C) 2013 by the authors; licensee MDPI, Basel, Switzerland. This article is an open access article distributed under the terms and conditions of the Creative Commons Attribution license (http://creativecommons.org/licenses/by/3.0/). 